\title{
Simulação acústica de uma sala multiúso para a prática musical: possibilidades de adaptação ao uso
}

\author{
Acoustic simulation of a multipurpose room for musical \\ practice: possibilities of adaptation to use
}

\section{Pollyanna Rodrigues Lima Erasmo Felipe Vergara}

\section{Resumo}

$\mathbf{P}$

ara que o músico desfrute de uma experiência satisfatória no ambiente de sua prática musical, um projeto acústico específico é essencial. Em um contexto em que cada vez mais um mesmo ambiente é utilizado para usos que requerem projetos acústicos distintos, as mesmas salas utilizadas para a palavra falada têm abrigado também a prática musical. O objetivo principal do trabalho foi demonstrar a contribuição da simulação computacional acústica na possibilidade de adaptação de uma sala de aula multiúso existente para o ensino e prática musical. Os principais parâmetros acústicos da sala foram obtidos experimentalmente, e, posteriormente, diferentes alternativas foram testadas fazendo uso da simulação computacional por meio do software Odeon, de forma que se obtivesse um modelo que adequasse a sala à prática musical. Esse modelo passou por diversas adaptações até que resultou em uma sala com valores de parâmetros acústicos dentro dos limites desejáveis, sendo, nas bandas de frequência de oitava de $500 \mathrm{~Hz}$ a $1.000 \mathrm{~Hz}$, TR 1,25 s; EDT 1,25 s; C80 1,98 dB; $\mathrm{D}_{50}$ 56,1\%; e STI 0,56. O modelo computacional resultante permitiu adaptar a sala multiúso, modelada para diferentes realidades, para a prática musical, considerando nesse modelo a inserção ou a remoção de materiais e dispositivos acústicos.

Palavras-chave: Prática musical. Sala multiúso. Simulação computacional.

Pollyanna Rodrigues Lima Centro Universitário Estácio de Santa

São J osé - SC - Brasil

Erasmo Felipe Vergara Universidade Federal de Santa Catarina Florianópolis - SC - Brasil

Recebido em 24/11/17 Aceito em 20/03/18

\begin{abstract}
For the musician to enjoy a satisfactory experience in his/her musical practice environment a specific acoustic design is essential. Within a context where the same environmentis increasingly beings used for uses that require distinct acoustic projects, the same rooms used for spoken word have also been used for musical practice. The main objective of this work was to demonstrate the contribution of computational acoustics simulation within the possibility of adaptation of an existing multipurpose classroom for teaching and musical practice. The main acoustic parameters of the room were obtained experimentally and later, different alternatives were tested using the computer simulation using Odeon software in order to obtain a model that suited the room for musical practice. This model underwent several adaptations until it resulted in a room with values of acoustic parameters within the desirable limits, being, in the octave frequency bands of 500 to $1000 \mathrm{~Hz}$ : TR 1.25 s; EDT 1.25 s; $C_{80} 1.98 \mathrm{~dB}$; $D_{50} 56.1 \%$; STI 0.56. The resulting computational model allowed to adapt the multipurpose room, modeled for different realities, for musical practice considering in this model the insertion or removal of acoustic materials and devices.
\end{abstract}

Keywords: Musical practice. Multipurpose room. Computer simulation. 


\section{Introdução}

A experiência satisfatória do usuário em determinado ambiente está associada a diversos fatores, tanto objetivos - qualidade do ambiente para a atividade que será vivenciada -, quanto subjetivos - experiências anteriores do usuário que permitam a diferentes usuários vivenciar um mesmo ambiente por perspectivas distintas. No que diz respeito ao ambiente adequado para o ensino e prática musical, um projeto acústico específico é essencial para a performance do músico, além dos requisitos mínimos de projeto, como conforto térmico, lumínico, ergonômico e de acessibilidade.

O contexto atual na arquitetura tem apresentado projetos cada vez mais multifuncionais, o que pode vir a prejudicar a sonoridade e a qualidade da performance artística. As mesmas salas utilizadas para a palavra falada cada vez mais têm abrigado também a prática musical, porém essas atividades exigem requisitos acústicos distintos, sendo que o projeto acústico da sala deve-se articular com suas características arquitetônicas (TAKAHASHI; BERTOLI, 2012). No caso de edificações já existentes, é preciso indicar soluções práticas e adaptáveis para que o ambiente seja adequado à atividade a qual se destina.

Para avaliar o desempenho acústico de salas, de modo geral, são usados os parâmetros de ruído de fundo e tempo de reverberação. O método tradicional para a medição do tempo de reverberação baseia-se na medida do decaimento do som no ambiente a partir da interrupção de um sinal sonoro (ruído branco ou rosa) emitido por uma fonte (INTERNATIONAL..., 2003; LONG, 2011; KUTTRUFF, 2009). Um método experimental mais recente para avaliar os parâmetros acústicos de uma sala baseia-se no princípio da resposta acústica ao impulso de um ambiente (INTERNATIONAL..., 2009), que permite que outros parâmetros, além do tempo de reverberação, sejam obtidos e relacionados com a clareza da música e a inteligibilidade da fala (BRADLEY, 2011).

Outra metodologia válida e confiável para caracterizar um ambiente acústico é por meio de simulação computacional. Um procedimento desse tipo leva em consideração a geometria da sala, a distribuição e as propriedades acústicas dos materiais, bem como a posição real da fonte emissora e dos receptores (VORLÄNDER, 1995; GADE, 2007; TOPA et al., 2011). Como resultado, é possível predizer os valores para diversos parâmetros acústicos importantes (tempo de reverberação (TR), tempo de decaimento inicial (EDT), clareza $\left(\mathrm{C}_{80}\right)$, definição $\left(\mathrm{D}_{50}\right)$, etc.) e vinculados ao decaimento da energia sonora no interior do recinto (TENENBAUM et al., 2007; VERGARA, 2013).

A simulação acústica de salas é muito útil como ferramenta de projeto para engenheiros e arquitetos, e vem ganhando cada vez mais importância e aplicações devido à maior precisão nos resultados encontrados nos diversos programas computacionais desenvolvidos. A possibilidade de se realizarem inúmeras modelagens com rapidez permite que as dimensões e as geometrias das salas sejam alteradas, bem como as propriedades acústicas dos materiais que as compõem, o que possibilita simular diferentes campos acústicos e prever os parâmetros acústicos envolvidos com a qualidade sonora desejada para a sala (VÖRLANDER; SUMMERS, 2008).

Projetos acústicos de intercomparação computacional, denominados de Round Robin, têm sido desenvolvidos com participantes e desenvolvedores de softwares de acústica de salas de diferentes países (VORLÄNDER, 1995; BORK, 2000, 2005). Nesses projetos tem sido usada uma sala de referência, e as equipes participantes computam parâmetros acústicos como TR, EDT, $\mathrm{C}_{80}$ e $\mathrm{D}_{50}$. Os bons resultados dessas comparações auxiliam os desenvolvedores de softwares e mostram o amadurecimento e o grande potencial das ferramentas de simulação computacional no projeto de acústica de salas.

Para que um modelo computacional se aproxime do modelo real de uma sala a ser simulada, o recomendado é comparar os dados dos parâmetros acústicos (TR, EDT, $\mathrm{C}_{80}$, $\mathrm{D}_{50}$ e outros), observados de medições, com os dados obtidos por meio de simulação computacional. O critério que mensura a diferença aceitável entre o modelo computacional e o modelo real recebe o nome de diferença apenas perceptível (just noticeable difference, JND) (INTERNATIONAL..., 2009; CHRISTENSEN; KOUTSOURIS, 2013). Christensen e Koutsouris (2013) observaram que a JND fornece uma boa sugestão quanto à precisão desejável em uma simulação computacional. Se a diferença entre o "real” (medição com precisão) e o parâmetro acústico simulado for menor do que a JND, então não há diferença perceptível, e o resultado da simulação é tão bom quanto deveria ser. Os valores de JND para alguns parâmetros de avaliação da qualidade acústica de salas estão compilados na Tabela 1 (VORLÄNDER; SUMMERS, 2008; INTERNATIONAL..., 2009; QUEIROZ DE SANT'ANA; ZANNIN, 2011; CHRISTENSEN; KOUTSOURIS, 2013). 
Dos parâmetros acústicos apresentados na Tabela 1, o tempo de reverberação (TR) é considerado o mais importante no estudo de espaços internos porque representa o tempo, em segundos, em que um som se apresenta audível em um ambiente após a fonte ser desligada (BISTAFA, 2006). Tempos de reverberação entre 0,3 s e 1,2 s são adequados para salas utilizadas para prática e ensino musical com volume menor que $400 \mathrm{~m}^{3}$ (LANE; MIKESKA, 1955; KARSAI, 1974; LAMBERTY, 1980; NAGATA, 1989; COHEN, 1992; TEUBER; VÖELKER, 1993; AMERICAN..., 2002; BUILDING..., 2003; LOKKI; SALMENSAARI, 2007; RYHERD, 2008). O tempo de decaimento inicial (EDT) e a clareza $\left(\mathrm{C}_{80}\right)$ são considerados de maior importância para a música. O EDT, em segundos, pode ser considerado subjetivamente mais importante do que o TR, pois é o parâmetro que descreve a percepção do tempo de reverberação pela orelha humana (INTERNATIONAL..., 2009). Já a $\mathrm{C}_{80}$, apresentada em decibels, representa o grau de clareza com que uma música tocada em uma sala é ouvida e demonstra o quão limpo e preciso um som se apresenta na sala (FIGUEIREDO, 2005). Quando C $_{80}$ não está adequada, o som se apresenta confuso (BERANEK 1996, 2004; SÁ, 2010; VERGARA; MARROS; PAUL, 2017).

No que diz respeito à qualidade de salas para a prática musical, Beranek (1996, 2004) publicou estudos em que avalia parâmetros - objetivos e subjetivos - associados à prática musical, buscando com músicos experientes e/ou maestros de música critérios que possam ser estabelecidos para garantir um espaço adequado à prática, especialmente no que diz respeito a grandes salas de concerto volumes superiores a $2.000 \mathrm{~m}^{3}$. Em publicação mais recente, Vergara, Marros e Paul (2017) apresentaram dados de TR, EDT, $\mathrm{C}_{80}$ e $\mathrm{D}_{50}$ de salas de prática e ensino de música com volume menor que $350 \mathrm{~m}^{3}$, tendo comparado os dados das salas obtidos com a opinião dos músicos que as utilizaram. Uma das salas preferidas pelos músicos foi uma sala de estudo individual com volume em torno de $80 \mathrm{~m}^{3}$ e com tempo de reverberação de 0,4 $\mathrm{s}$, descrita como seca por eles. Outra sala preferida foi uma sala de estudo de aula coletiva com volume em torno de $330 \mathrm{~m}^{3}$ e tempo de reverberação de 1,4 $\mathrm{s}$, caracterizada como reverberante pelos músicos.

Neste estudo, uma sala multiúso destinada ao ensino e prática musical de uma orquestra foi o objeto de uma avaliação da qualidade dos parâmetros acústicos determinados mediante ensaios em campo e pela simulação computacional. Os ensaios e as simulações seguiram as recomendações da norma ISO 3382 para determinar TR, EDT, $\mathrm{C}_{80}$ e $\mathrm{D}_{50}$. A partir da caracterização acústica da sala multiúso, o artigo demonstra a contribuição da simulação computacional acústica na possibilidade de adaptação de uma sala de aula multiúso para o ensino e prática musical.

\section{Método}

A sala multiúso, aqui caracterizada como de artes visuais, é uma das três salas de aula artísticas de uma instituição de ensino médio e superior localizada em Florianópolis, SC. As salas de música, de artes visuais e de teatro são utilizadas para o ensino e prática musical, atualmente maior demanda da coordenadoria artística da instituição. As atividades voltadas à música são requeridas pelo Curso Básico de Instrumentos de Orquestra de qualificação profissional, pelos ensaios da Orquestra Experimental e do Coral da instituição, bem como pelas aulas teóricas e práticas de música para os alunos do ensino médio conforme as exigências da Lei no 11.769 (BRASIL, 2008).

A sala possui formato retangular, com uma área de $59,5 \mathrm{~m}^{2}$ e volume de $204,7 \mathrm{~m}^{3}$, e é utilizada para todos os tipos de atividades artísticas pelos docentes e alunos da instituição. Frequentemente vem sendo empregada para a prática musical. Possui duas portas de acesso, do tipo giro de uma folha, e no sentido oposto a elas há oito janelas do tipo maximar. As Figuras 1 e 2 mostram o interior da sala multiúso de artes visuais. Tratando-se do uso para a música, a sala não possui uma ocupação regular pelos músicos, tendo em vista que é utilizada para o estudo musical individualmente, por um músico, ou por dez músicos durante o ensaio. Os principais instrumentos utilizados por esses músicos são violinos, contrabaixo e piano.

Tabela 1 - Diferença apenas perceptível (J ND) da impressão subjetiva acústica de salas

\begin{tabular}{c|c|c|c}
\hline $\begin{array}{c}\text { Aspecto } \\
\text { subjetivo }\end{array}$ & $\begin{array}{c}\text { Parâmetro } \\
\text { objetivo }\end{array}$ & Frequência média (Hz) & JND \\
\hline Reverberação & TR (s) & 500 a 1.000 & $5 \%$ \\
Percepção da reverberação & EDT (s) & 500 a 1.000 & $5 \%$ \\
Clareza & $\mathrm{C}_{80}$ & 500 a 1.000 & $1 \mathrm{~dB}$ \\
Definição & $\mathrm{D}_{50}$ & 500 a 1.000 & $5 \%$ \\
\hline
\end{tabular}

Fonte: Vorländer e Summers (2008), International Organization for Standardization (2009), Queiroz de Sant'Ana (2011) e Christensen e Koutsouris (2013). 
Figura 1 - Interior da sala de artes visuais

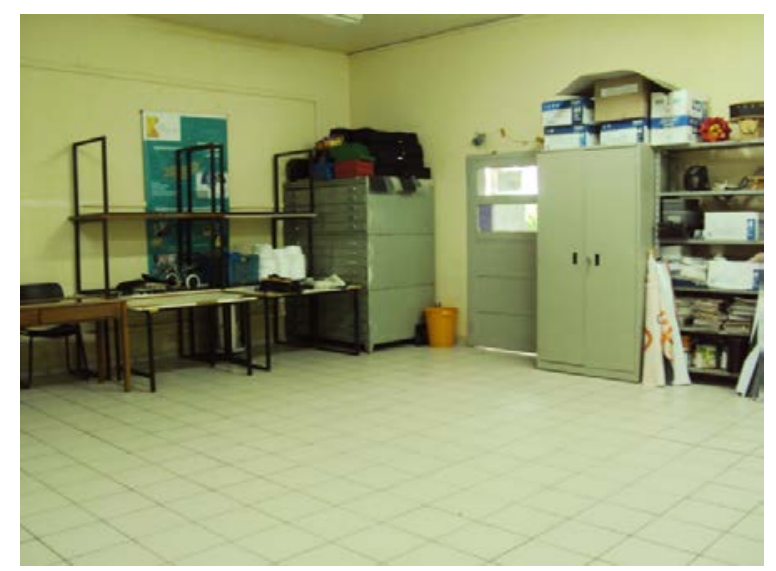

Figura 2 - Interior da sala de artes visuais

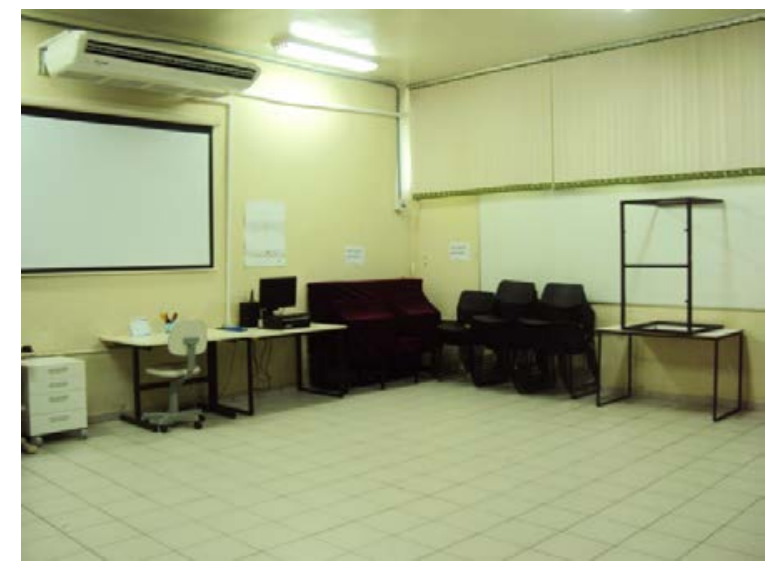

Em relação aos materiais das superfícies, as paredes são simples, de alvenaria com tijolos cerâmicos furados, rebocadas e pintadas com tinta látex acrílica. O piso é de cerâmica. O teto não possui forro, sendo apenas de reboco alisado e pintado com tinta látex acrílica. As janelas são de estrutura de PVC e vidro simples de $6 \mathrm{~mm}$. As portas são de madeira maciça com duas janelas de vidro cada uma. Persianas cobrem toda a extensão das janelas de vidro. A Tabela 2 apresenta os materiais que compõem as superfícies da sala, bem como suas áreas e coeficientes de absorção sonora.

O mobiliário da sala de artes visuais é muito variado, como mesa em MDF, estantes e armários metálicos, mapoteca metálica, pia para lavação de materiais, pranchetas para desenhos, telão para projeções, cadeiras plásticas e estofadas, piano de parede, violinos e outros materiais para atividades artísticas. Os coeficientes de absorção sonora adotados na simulação computacional para cada material e objeto são apresentados na seção Modelagem computacional da sala multiúso Ajuste do modelo.

\section{Avaliação dos parâmetros acústicos da sala multiúso}

A determinação dos parâmetros acústicos da sala multiúso de artes visuais foi baseada na medição acústica da resposta ao impulso da sala (room impulse response - RIR) de acordo com as recomendações da norma ISO 3382-1 (INTERNATIONAL..., 2009). Os parâmetros acústicos resultantes dessa medição foram tempo de reverberação (TR), tempo de decaimento inicial (EDT), clareza (C80), definição (D50) e índice de transmissão da fala (STI).

O sistema utilizado para a medição da RIR foi o sonômetro Solo da marca $01 \mathrm{~dB}$, um computador portátil com o programa de aquisição e pósprocessamento dBBati32, e balões de látex de tamanho gigante como fonte sonora impulsiva. $\mathrm{O}$ sonômetro Solo foi devidamente calibrado no momento da medição com o calibrador acústico 01dB-Stell (94 dB, ref. 0,00002 Pa, $1.000 \mathrm{~Hz}$ ). As medições foram efetivadas pela combinação de duas posições de fontes e quatro pontos receptores, sendo em cada ponto receptor realizadas três medições. A fonte sonora foi acionada a uma altura 
de 1,5 m do piso, e o microfone do sonômetro, em cada um dos pontos receptores, a 1,2 $\mathrm{m}$ do piso. A Figura 3 mostra a planta baixa da sala com a posição das duas fontes 1 e 2 (F1 e F2) e dos pontos receptores da fonte $1(\mathrm{P} 11, \mathrm{P} 12, \mathrm{P} 13, \mathrm{P} 14)$ e da fonte 2 (P21, P22, P23, P24).

Antes de efetuar as medições acústicas, as cadeiras da sala foram removidas tendo em vista que esse mobiliário não permanece fixo na sala. A remoção dessas cadeiras permitiu a diminuição da complexidade do modelo geométrico e computacional a ser confeccionado na etapa de simulação computacional da sala multiúso. Além disso, as persianas foram mantidas fechadas.

\section{Modelagem computacional da sala multiúso}

Para estudar o comportamento acústico de uma sala a partir da predição acústica com um programa computacional são necessárias informações de suas características geométricas, das propriedades de absorção e espalhamento sonoro das superfícies, assim como das características de potência sonora e de diretividade da fonte que geraria o som. É a partir de uma simulação acústica de uma sala que é possível predizer sua resposta impulsiva (RIR) num modelo tridimensional e obter para diferentes pontos receptores parâmetros acústicos como tempo de reverberação, tempo de decaimento inicial, distribuição espacial de níveis de pressão sonora, claridade, definição e índice de transmissão da fala.

Tabela 2 - Materiais e coeficientes de absorção sonora da sala de artes visuais

\begin{tabular}{|c|c|c|c|c|c|c|c|}
\hline \multirow{3}{*}{ Material } & \multirow{3}{*}{$\begin{array}{c}\text { Área } \\
\left(\mathbf{m}^{2}\right)\end{array}$} & \multicolumn{6}{|c|}{ Coeficiente de absorção } \\
\hline & & 125 & 250 & 500 & 1.000 & 2.000 & 4.000 \\
\hline & & \multicolumn{6}{|c|}{ (Hz) } \\
\hline Piso cerâmico* & 59,11 & 0,01 & 0,01 & 0,01 & 0,02 & 0,02 & 0,02 \\
\hline Teto rebocado liso** & 59,52 & 0,02 & 0,02 & 0,03 & 0,04 & 0,04 & 0,03 \\
\hline Parede de alvenaria rebocada** & 91,65 & 0,03 & 0,03 & 0,04 & 0,04 & 0,04 & 0,03 \\
\hline Portas de madeira fechadas** & 4,26 & 0,24 & 0,19 & 0,14 & 0,08 & 0,13 & 0,10 \\
\hline Persiana** & 10,20 & 0,03 & 0,04 & 0,11 & 0,17 & 0,24 & 0,35 \\
\hline
\end{tabular}

Fonte: *Carvalho (2010) e**Bistafa (2006).

Figura 3 - Planta baixa da sala de artes visuais indicando a locação das fontes sonoras e os pontos receptores da medição

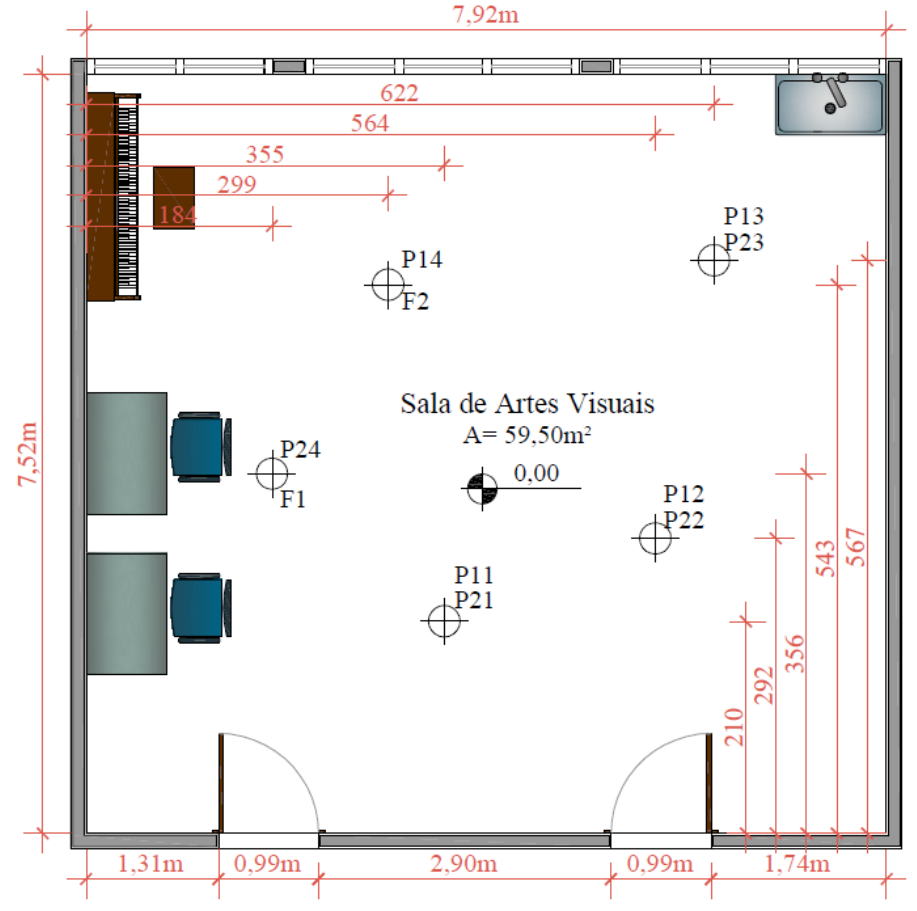


Neste estudo foi empregado o programa computacional Odeon, desenvolvido pelo Departamento de Tecnologia Acústica da Universidade Técnica da Dinamarca. O programa utiliza o método híbrido para realizar as simulações, aplicando às primeiras reflexões o método da fonte imagem modificada, e às reflexões tardias, o método das fontes secundárias. O método híbrido é bastante confiável, mas a limitação está no aumento da ordem de reflexões sonoras, o que implica maior tempo de processamento dos cálculos. Por essa razão é que somente são aproveitadas as primeiras reflexões. Além disso, o programa simula as perdas por dispersão (espalhamento), criando tanto reflexões especulares quanto reflexões difusas. A confiabilidade dos resultados dependerá dos valores definidos durante a simulação para o número de raios, a duração da resposta impulsiva, a ordem de transição, a máxima ordem de reflexão e as simplificações do modelo do local. Neste estudo foram modeladas as superfícies como planas, número de raios igual a 1.000, duração da resposta impulsiva igual a 4.000 ms, resolução da resposta impulsiva igual a 3,0 ms, ordem de transição igual a 2, ordem de reflexão igual a 1.000, número de raios de primeiras reflexões igual a 100, e densidade de reflexão tardia desejada igual a $10.000 \mathrm{~ms}$ (CHRISTENSEN; KOUTSOURIS, 2013).

Essa etapa de simulação foi realizada em quatro fases principais para construir o modelo geométrico e verificar os parâmetros acústicos (TR, EDT, $\mathrm{C}_{80}$, $\mathrm{D}_{50}$ e STI) nas bandas de frequências de oitava entre $125 \mathrm{~Hz}$ e $4.000 \mathrm{~Hz}$, obtidos pela medição da RIR na sala multiúso. As etapas do processo de modelagem foram confecção do modelo geométrico, definição e aplicação de materiais, ajuste do modelo e simulação acústica. A Figura 4 ilustra de maneira esquemática os processos que envolveram a etapa de simulação computacional.

\section{Confecção do modelo geométrico}

A criação de um arquivo contendo as informações das características geométricas da sala pode ser por meio da digitação dos dados em um arquivo de texto no módulo Odeon editor (Editor do Odeon ${ }^{1}$ ), utilizando o próprio programa computacional ou o plugin disponível para o SketchUp, ou importando arquivos criados em programas de CAD (por exemplo, AutoCAD, Autodesk Revit, 3DStudioMax) com extensão “.dxf”, ou, ainda, combinando esses diferentes métodos de modelagem (CHRISTENSEN; KOUTSOURIS, 2013). O método adotado para este trabalho foi a confecção do modelo no SketchUp por se mostrar um método menos complexo e mais rápido de implementar.

A geometria da sala foi modelada de maneira simplificada, com a minimização de superfícies, desconsiderando pequenas bordas, reentrâncias e detalhes irrelevantes para a simulação, seguindo o levantamento arquitetônico da sala. No modelo da sala optou-se por gerar a geometria sem a inserção dos mobiliários e objetos para evitar arquivos pesados e processos de simulação muito demorados. Cada tipologia de superfície foi modelada em uma layer, ou camada, diferente, segundo o material de composição, para posterior inserção de dados de coeficiente de absorção sonora de acordo com os valores da Tabela 2. Na sequência, o modelo geométrico foi exportado na extensão “.par” usando o Sketchup plugin for ODEON (plugin Sketchup para ODEON ${ }^{2}$ ).

Figura 4 - Procedimentos e ferramentas envolvidos durante a modelagem acústica da sala multiúso

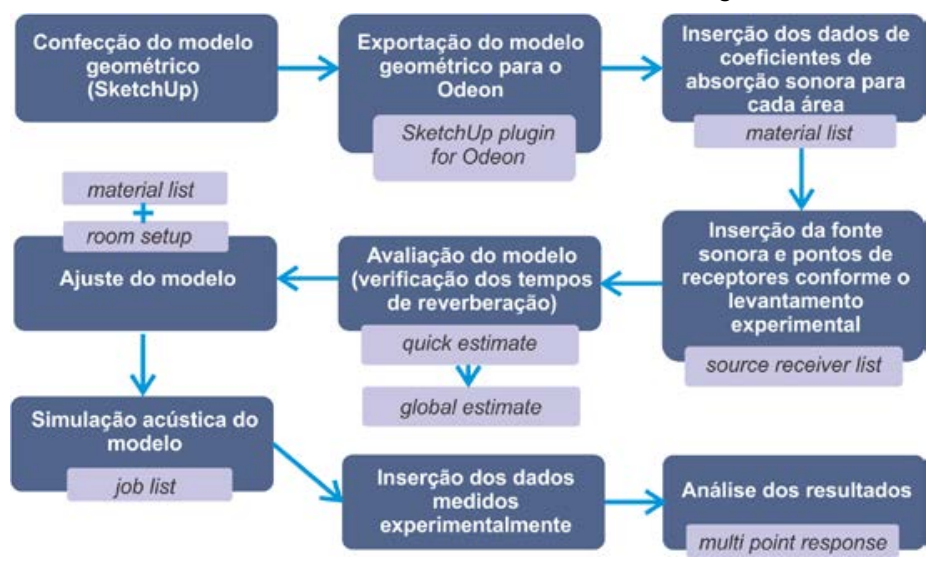

1Tradução dos autores.

${ }^{2}$ Tradução dos autores. 


\section{Definição e aplicação de materiais}

Após a verificação do modelo geométrico de formato “.par”, os coeficientes de absorção sonora de cada superfície foram inseridos para cada camada diferente de acordo com o material. A ferramenta "material list" (lista de materiais ${ }^{3}$ ) e a opção "edit material list”" (editar lista de materiais²) permitiram a seleção e a inserção desses coeficientes por banda de frequência de oitava entre $63 \mathrm{~Hz}$ e $8.000 \mathrm{~Hz}$. A inclusão e a localização da fonte sonora bem como dos pontos de recepção do sinal foram realizadas com o recurso "sourcerecives list” (lista de fontes-receptores ${ }^{4}$ ), em que é inserida tanto a posição da fonte quanto a dos receptores, indicando as coordenadas $x, y$ e $z$ de localização de cada um desses elementos de acordo com as localizações deles nas medições acústicas da RIR da sala multiúso.

\section{Ajuste do modelo}

Existem dois métodos para a verificação inicial da qualidade do modelo geométrico e da aplicabilidade dos materiais escolhidos no Odeon. O primeiro utiliza o modo "quick estimate" (estimativa rápida $\left.{ }^{2}\right)$, e o segundo, o "global estimate" (estimativa global²). Em cada um deles é calculado o tempo de reverberação do modelo criado (CHRISTENSEN; KOUTSOURIS, 2013).

A “quick estimate” está baseada nas fórmulas de Sabine, Eyring e Arau-Puchades para determinar os respectivos tempos de reverberação e assume a condição de campo sonoro difuso para a sala simulada. A “global estimate” é uma metodologia mais precisa de verificação do TR porque não são assumidas suposições sobre o campo difuso, o que resulta em um TR mais confiável, desde que sejam inseridos os coeficientes de dispersão/espalhamento sonoro dos materiais (CHRISTENSEN; KOUTSOURIS, 2013). Apesar do potencial de confiabilidade da "global estimate", a escassa informação dos devidos coeficientes de dispersão/espalhamento sonoro dos materiais, conforme já observado em Rindel (2000), resultou em uma limitação inicial para essa simulação, de forma que esses dados não foram inseridos, reduzindo, assim, o potencial de confiabilidade desse método. No entanto, ambos os métodos foram utilizados para a verificação inicial da qualidade do modelo, o que resultou em grande discrepância dos tempos de reverberação obtidos pela simulação com aqueles da medição acústica da RIR.
O modelo em desenvolvimento passou por ajustes para minimizar a diferença entre os dados dos parâmetros acústicos da medição e os da simulação. Para isso, foram feitas alterações nas configurações da sala multiúso com a ferramenta "room setup" (configuração da sala²), conforme sugeria o manual do usuário do Odeon. Alteraram-se, então, o número de raios, de 1.000 para 5.000 raios, a ordem de reflexão, de 1.000 para 2.000 , e a densidade de reflexão tardia desejada, de 10.000 ms para 999.999 ms. Ainda assim, o modelo computacional da sala de artes visuais pouco se aproximou do real, possivelmente pela grande variedade de elementos presentes na sala real, que foram desconsiderados no modelo computacional inicial.

Diante da não compatibilidade dos resultados do modelo computacional com os das medições em campo, optou-se por inserirem-se no modelo geométrico da simulação os elementos existentes na sala que, conhecendo seus coeficientes de absorção, apresentavam área e coeficientes de absorção sonora relevantes, aqui considerados maiores que 0,10 , conforme mostra a Tabela 3 . Esses elementos foram as mesas de madeira, os pianos e as estantes de livros, bem como duas pessoas que estavam presentes durante a execução das medições acústicas. Para inserir simplificadamente duas pessoas no modelo geométrico utilizou-se a referência de Silva (2016), que afirmou que a área da superfície do corpo humano considerado normal é de 1,6 $\mathrm{m}^{2}$ para mulheres e de 1,9 $\mathrm{m}^{2}$ para homens aproximadamente.

\section{Simulação acústica}

A próxima etapa do processo de simulação foi configurar o tipo de pós-processamento dos resultados da RIR no modelo computacional usando a opção "job list" (lista de trabalhos ${ }^{5}$ ). Existem três formas de obtenção dos resultados: "single point response” (resposta de ponto único ${ }^{6}$ ), "multi point response" (resposta de múltiplos pontos ${ }^{3}$ ) e "grid response" (resposta de grade ${ }^{7}$ ). A primeira oferece um cálculo detalhado dos resultados para um único ponto de receptor selecionado; a segunda oferece os parâmetros acústicos da sala para todos os receptores definidos na lista de receptores; e a última oferece um cálculo geral dos parâmetros do quarto levando em conta uma grade criada no menu “define grid” (definir grade ${ }^{8}$ ) (CHRISTENSEN; KOUTSOURIS, 2013).

Inicialmente a opção escolhida foi a "single point response”, para obter dados detalhados de cada um

\footnotetext{
3Tradução dos autores.

${ }^{4}$ Tradução dos autores.

5Tradução dos autores.

${ }^{6}$ Tradução dos autores.
} 
dos pontos de recepção sonora. Posteriormente foi selecionado o tipo de resposta "multi point response", sendo possível inserir os dados das medições de campo e verificá-los com os dados da simulação. Após os devidos ajustes, já apresentados, o modelo computacional atingiu as recomendações da JND propostas pela ISO 33821:2009, conforme mostra a Tabela 4. Os valores dos parâmetros acústicos (TR, EDT, $\mathrm{C}_{80}$ e $\mathrm{D}_{50}$ ) para as bandas de frequências de $500 \mathrm{~Hz}$ e $1.000 \mathrm{~Hz}$, obtidos a partir das medições e da simulação da RIR, atenderam aos limites de JND.

As Figuras 5 e 6 apresentam uma comparação entre os resultados obtidos nas simulações e nas medições para os parâmetros considerados mais relevantes para a sensação do músico: sensação da reverberação (EDT) e clareza com que se percebe a música $\left(\mathrm{C}_{80}\right)$. Os valores médios de EDT e $\mathrm{C}_{80}$ obtidos por banda de frequência de oitava $(125 \mathrm{~Hz}$ a $4.000 \mathrm{~Hz}$ ) confirmam a proximidade do modelo computacional simulado com o modelo real.

\section{Resultados e discussão}

Após resultar em um modelo computacional mais próximo do real, e considerando que a sala de artes visuais não se encontra adequada para a prática musical por extrapolar a maioria dos valores desejados para os parâmetros indicados pela bibliografia, novas simulações foram realizadas para sugerir alternativas de adaptação da sala ao uso e prática musical.

Tabela 3 - Materiais e coeficientes de absorção sonora adicionados na sala de artes visuais

\begin{tabular}{|c|c|c|c|c|c|c|c|}
\hline \multirow{3}{*}{ Material } & \multirow{3}{*}{$\begin{array}{c}\text { Área } \\
\left(\mathbf{m}^{2}\right)\end{array}$} & \multicolumn{6}{|c|}{ Coeficiente de absorção sonora } \\
\hline & & 125 & 250 & 500 & 1.000 & 2.000 & 4.000 \\
\hline & & \multicolumn{6}{|c|}{ (Hz) } \\
\hline Pessoa de pé* & 3,20 & 0,11 & 0,20 & 0,27 & 0,26 & 0,28 & 0,23 \\
\hline Mesa de madeira** & 2,97 & 0,10 & 0,10 & 0,05 & 0,05 & 0,04 & 0,04 \\
\hline Piano** & 5,56 & 0,20 & 0,52 & 0,60 & 0,57 & 0,52 & 0,43 \\
\hline Estante de livros** & 9,48 & 0,11 & 0,33 & 0,90 & 0,60 & 0,79 & 0,68 \\
\hline
\end{tabular}

Fonte: *Bistafa (2006) e **Carvalho (2010).

Tabela 4 - Diferença entre os valores medidos e os simulados na faixa de $500 \mathrm{~Hz}$ e $1.000 \mathrm{~Hz}$, comparados com a J ND

\begin{tabular}{c|c|c|c|c}
\hline & TR & EDT & $\begin{array}{c}\mathbf{C}_{80} \\
{[\mathbf{d B}]}\end{array}$ & $\begin{array}{c}\mathbf{D}_{\mathbf{5 0}} \\
\mathbf{( \% )}\end{array}$ \\
\hline Medição & $1,86 \mathrm{~s}$ & $1,89 \mathrm{~s}$ & $-1,42$ & 31,9 \\
Simulação & $1,93 \mathrm{~s}$ & $1,95 \mathrm{~s}$ & $-0,86$ & 32,3 \\
Diferença & $4 \%$ & $3 \%$ & 0,56 & 1,2 \\
JND & $5 \%$ & $5 \%$ & 1 & 5 \\
\hline
\end{tabular}

Figura 5 - Comparativo do tempo de decaimento inicial (EDT) das medições e da simulação para a sala de artes visuais

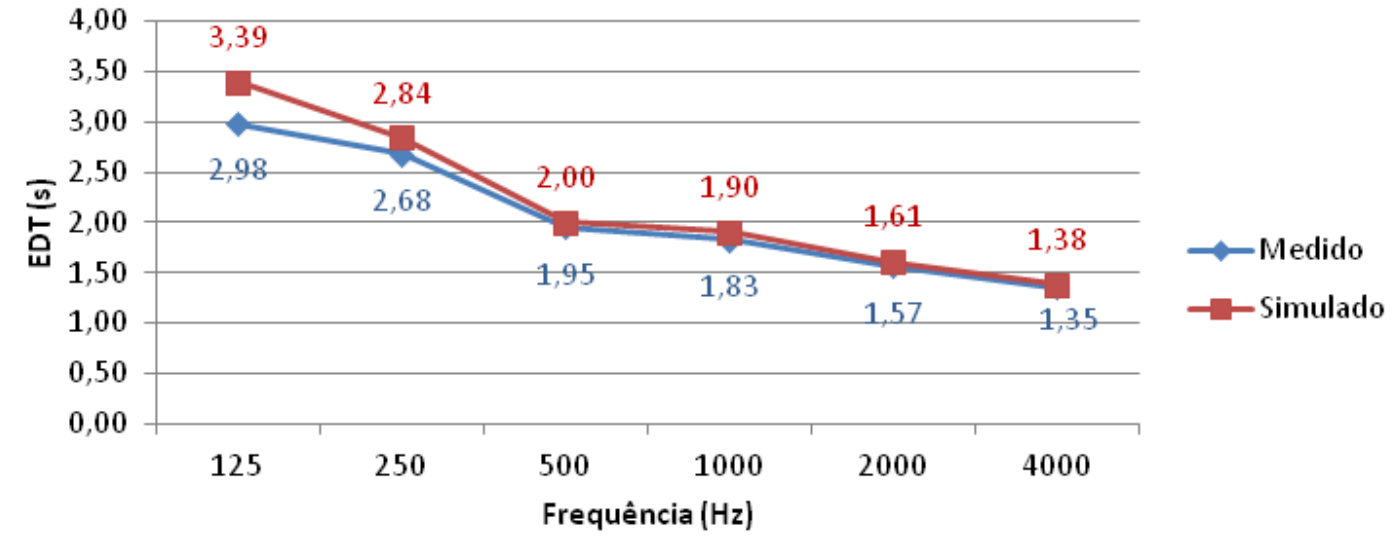




\section{Figura 6 - Comparativo da clareza $\left(\mathrm{C}_{80}\right)$ das medições e da simulação para a sala de artes visuais}

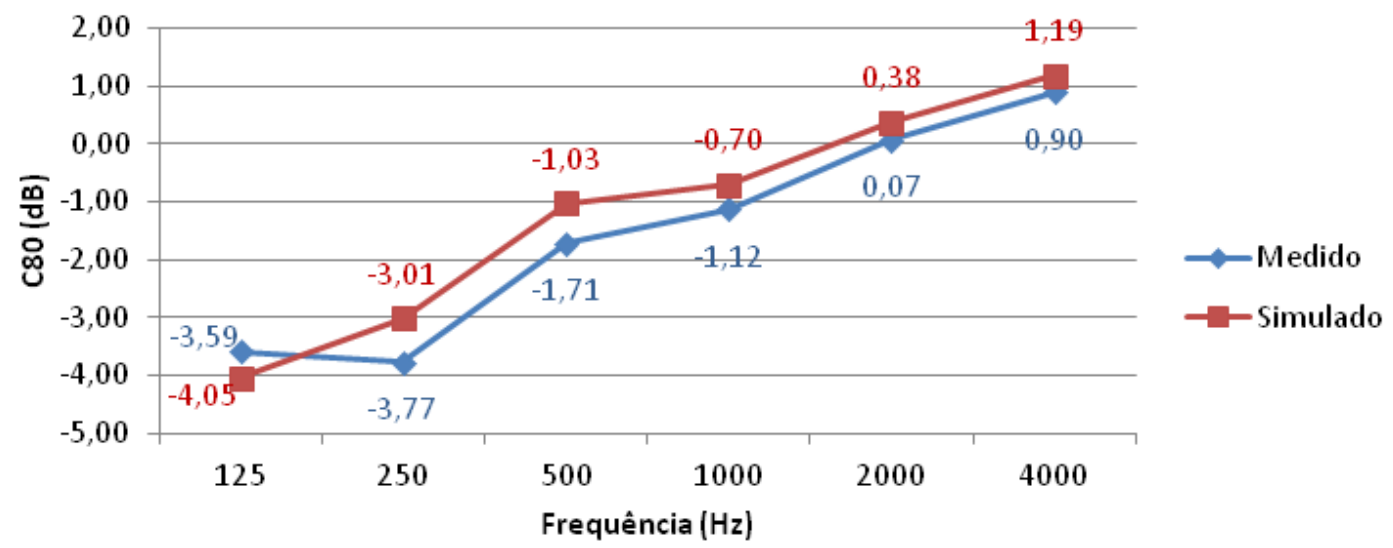

Antes de iniciarem-se as simulações, os valores dos parâmetros acústicos da simulação foram compilados em face dos valores desejados para a prática musical indicados em Beranek (1996, 2004), Custódio (2004), Sá (2010) e Vergara, Marros e Paul (2017), conforme pode ser visto na Tabela 5. Além dos parâmetros acústicos obtidos pela simulação, outros três parâmetros considerados importantes para a música foram calculados: vivacidade, brilho e calor (BERANEK, 2004). Estes três parâmetros representam o equilíbrio da música nas frequências médias (500 Hz e $1.000 \mathrm{~Hz}$ ), altas $(2.000 \mathrm{~Hz}$ e $4.000 \mathrm{~Hz})$ e baixas $(125 \mathrm{~Hz}$ e $250 \mathrm{~Hz})$ respectivamente. Os valores dos parâmetros TR, EDT, $C_{80}$ e $D_{50}$ são representativos da média entre as bandas de frequência de oitava de $500 \mathrm{~Hz}$ e 1.000 Hz. A recomendação de valores para o calor considera que o TR seja menor ou igual a 1,8 s (BERANEK, 1996).

A sala de artes visuais apresentou valores relativamente próximos daqueles considerados adequados para a prática musical. Nesse caso, supõe-se que isso se deve ao fato da existência de diversos elementos absorvedores sonoros de som na sala, como prateleiras e estantes com livros e materiais diversos para artes plásticas. Ainda que exista alguma proximidade dos valores dos parâmetros encontrados nessa sala com aqueles considerados adequados para a prática musical, seriam necessárias algumas adaptações para que esses valores se adequassem aos limites desejados.

Uma limitação para a inserção de dispositivos para corrigir esses parâmetros nessa sala foi a pouca área disponível nas paredes para fixação de algum dispositivo, especialmente pelos dois quadros que ocupam grande parte de duas paredes, pela utilização das paredes na projeção de vídeos e, ainda, pelas estantes para armazenamento de materiais, pelas mesas e por outros elementos. Diante dessa limitação inicial, uma primeira alternativa foi a inserção de um tapete para aumentar consideravelmente a absorção sonora do piso. O resultado foi satisfatório para que o TR e outros parâmetros se aproximassem ainda mais dos limites desejados, mas o cálculo do parâmetro calor, com base nos valores de TR da simulação, ainda se encontrava distante do desejado, o que significava que a sensação do TR nas frequências baixas encontrava-se irregular para a sensação do TR das frequências médias. O TR nas baixas frequências estava ainda muito alto quando comparado ao TR das médias frequências.

A alternativa adotada para corrigir essa irregularidade foi utilizar dispositivos chamados de bass traps, que servem para controlar os sons graves dentro de salas e são capazes de absorver consideravelmente o som nas baixas frequências (de $63 \mathrm{~Hz}$ a $200 \mathrm{~Hz}$ ). Uma boa sugestão de localização desses dispositivos é entre parede e teto, o que foi ao encontro da limitação de área de parede existente nessa sala. O dispositivo bass trap Vicoustic Super Bass Extreme foi inserido entre teto e parede, na parede de acesso da sala, por toda a extensão.

Após algumas tentativas e ajustes, o modelo da simulação com as alternativas adotadas resultou em valores de parâmetros acústicos dentro dos limites desejáveis para a prática musical, modelo esse apresentado nas especificações descritas na Figura 7 e na Tabela 6.

Com as adaptações, apenas o parâmetro vivacidade não ficou dentro do recomendado por Beranek (2004), entretanto cabe ressaltar que o autor tratava de vivacidade adequada para salas de concerto, com volumes muito superiores - acima de $2.000 \mathrm{~m}^{3}$ - aos de pequenas salas de prática - inferiores a $300 \mathrm{~m}^{3}$. Dessa forma, considerou-se que, estando as salas dentro dos limites desejados para o TR indicado para pequenas salas de prática musical, conforme Sá (2010) e Vergara, Marros e Paul (2017), entre $0,4 \mathrm{~s}$ e $1,4 \mathrm{~s}$, consequentemente a vivacidade também estaria adequada para essa tipologia. 
Tabela 5 - Valores dos parâmetros acústicos simulados e valores desejados para os parâmetros de acústica de salas voltadas para a prática musical

\begin{tabular}{c|c|c|c}
\hline Parâmetro & Simulação & Desejado & Cálculo \\
\hline TR (s) & 1,93 & $0,4-1,4$ & -- \\
EDT (s) & 1,95 & $0,4-1,4$ & -- \\
C $_{80}(\mathrm{~dB})$ & $-0,86$ & $1-5$ & -- \\
D $_{50} \%$ & 31,5 & $50-65$ & -- \\
STI & 0,44 & $>0,45$ & -- \\
Vivacidade & -- & $>1,6$ & 1,9 \\
Calor & -- & $1,1-1,45$ & 1,5 \\
Brilho & -- & $>0,7$ & 0,77 \\
\hline
\end{tabular}

Figura 7 - Modelo geométrico e computacional adotado para a sala de artes visuais indicando os diferentes elementos

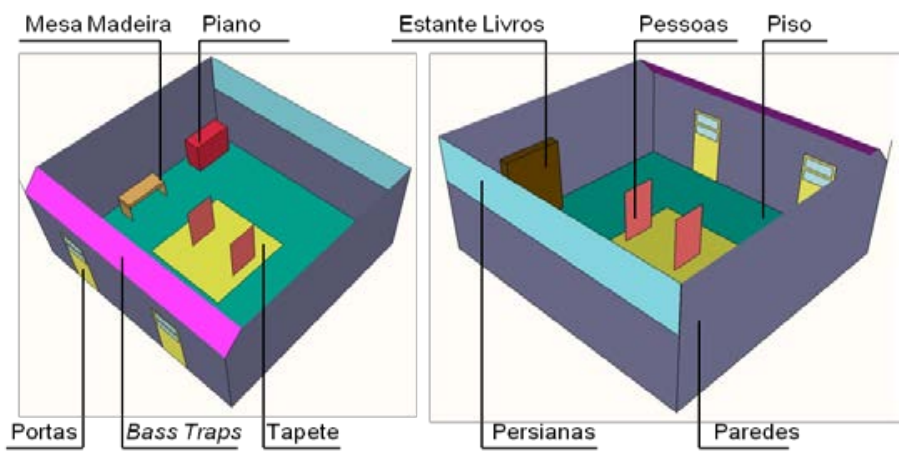

Tabela 6 - Especificações de materiais, áreas e coeficientes de absorção sonora usados na simulação da sala de artes visuais

\begin{tabular}{|c|c|c|c|c|c|c|c|}
\hline \multirow{3}{*}{ Material } & \multirow{3}{*}{ Área $\left(\mathbf{m}^{2}\right)$} & \multicolumn{6}{|c|}{ Coeficiente de absorção } \\
\hline & & 125 & 250 & 500 & 1.000 & 2.000 & 4.000 \\
\hline & & \multicolumn{6}{|c|}{ (Hz) } \\
\hline Piso cerâmico* & 50,01 & 0,01 & 0,01 & 0,01 & 0,02 & 0,02 & 0,02 \\
\hline Teto rebocado liso** & 54,77 & 0,02 & 0,02 & 0,03 & 0,04 & 0,04 & 0,03 \\
\hline Parede de alvenaria rebocada** & 86,64 & 0,03 & 0,03 & 0,04 & 0,04 & 0,04 & 0,03 \\
\hline Portas de madeira fechadas** & 4,26 & 0,24 & 0,19 & 0,14 & 0,08 & 0,13 & 0,10 \\
\hline Persiana** & 10,20 & 0,03 & 0,04 & 0,11 & 0,17 & 0,24 & 0,35 \\
\hline Pessoa de pé** & 3,20 & 0,11 & 0,20 & 0,27 & 0,26 & 0,28 & 0,23 \\
\hline Mesa de madeira* & 2,97 & 0,10 & 0,10 & 0,05 & 0,05 & 0,04 & 0,04 \\
\hline Piano* & 5,56 & 0,20 & 0,52 & 0,60 & 0,57 & 0,52 & 0,43 \\
\hline Estante de livro* & 9,48 & 0,11 & 0,33 & 0,90 & 0,60 & 0,79 & 0,68 \\
\hline Tapete com 5 mm de espessura ${ }^{+}$ & 9,00 & 0,04 & 0,04 & 0,15 & 0,29 & 0,52 & 0,59 \\
\hline Bass traps $^{++}$ & 6,72 & 0,61 & 0,39 & 0,28 & 0,43 & 0,43 & 0,32 \\
\hline
\end{tabular}

Fonte: *Carvalho (2010), **Bistafa (2006) e "Fabricante.

Os resultados obtidos no modelo computacional após as adaptações foram compilados na Tabela 7 , que apresenta esses valores simulados comparados com os valores indicados como adequados para a prática musical.

\section{Conclusões}

O trabalho permitiu verificar que salas de aula multiúso podem adequar-se à prática musical com algumas intervenções, desde que um apropriado estudo acústico seja realizado, que garanta padrões aceitáveis de qualidade ao uso para o qual a sala se destina. Além dos dispositivos acústicos disponíveis no mercado para atender às diferentes necessidades de uma sala - como foi o caso do bass trap neste trabalho -, elementos comuns de decoração ou utilidade, quando devidamente dimensionados, podem melhorar a resposta acústica de uma sala - como foi o caso do tapete neste estudo. 
Tabela 7 - Resultados obtidos nos modelos após adaptações comparados aos resultados desejados

\begin{tabular}{c|c|c|c}
\hline Parâmetro & Desejado & Simulação & Cálculo \\
\hline TR (s) & $0,4-1,4$ & 1,25 & -- \\
EDT (s) & $0,4-1,4$ & 1,25 & -- \\
C $_{80}(\mathrm{~dB})$ & $1-5$ & 1,98 & -- \\
D $_{50} \%$ & $50-65$ & 56,1 & -- \\
STI & $>0,45$ & 0,56 & -- \\
Vivacidade & $>1,6$ & -- & 1,25 \\
Calor & $1,1-1,45$ & -- & 1,36 \\
Brilho & $>0,7$ & -- & 0,81 \\
\hline
\end{tabular}

A simulação computacional possibilitou criar um modelo computacional para a sala, fiel ao real, flexível a testes de diferentes combinações de modelos adaptados da sala com o objetivo de propor aquele que melhor se adequasse às necessidades da prática musical, de forma a permitir a inserção e a remoção de materiais e dispositivos acústicos, conforme os resultados mostravam ser necessários ou não tais elementos. Com o modelo computacional resultante é possível adaptar a sala modelada para diferentes realidades, seja de prática musical ou qualquer outro uso que possa vir a ser dado a ela, considerando que com esse modelo podem testar-se diferentes possibilidades de inserção e/ou remoção de materiais acústicos. No caso da sala aqui estudada, ela pode receber as atividades de música ou atividades de fala em diferentes momentos, desde que os materiais e elementos, devidamente dimensionados e posicionados, sejam inseridos e removidos conforme a necessidade de uso.

\section{Referências}

\section{AMERICAN NATIONAL STANDARDS \\ INSTITUTE. ANSI S12.60-2002: acoustical performance criteria, design requirements, and guidelines for schools. Melville, 2002.}

BERANEK, L. Concert and Opera Halls: how they sound. New York: Acoustical Society of America, 1996.

BERANEK, L. L. Concert Halls and Opera House: music, acoustics \& architecture. $2^{\text {nd }}$ ed. New York: Springer, 2004.

BISTAFA, Sylvio R. Acústica Aplicada ao Controle de Ruído. São Paulo: Blucher, 2006.

BORK, I. A Comparison of Room Simulation Software: the 2nd Round Robin on Room Acoustical Computer Simulation. Acta Acustica, v. 86, p. 943-956, 2000.

BORK, I. Report on the 3rd Round Robin on Room Acoustical Computer Simulation - Part II: Calculations. Acta Acustica, v. 91, n. 4, p. 753763, 2005.
BRADLEY, J. Review of Objective Room Acoustics Measures and Future Needs. Applied Acoustics, v. 72, n. 10, p. 713-720, 2011.

BRASIL. Lei $\mathrm{n}^{\circ} 11.769$, de 18 de agosto de 2008, que altera a Lei no 9.394, de 20 de dezembro de 1996, Lei de Diretrizes e Bases da Educação, para dispor sobre a obrigatoriedade do ensino da música na educação básica. Diário Oficial [da]

Republica Federativa do Brasil, Brasília, 19 ago. 2008. Seção 1. p. 1.

BUILDING BULLETIN 93. Acoustic, Design of Schools: a design guide. London: Department for Education and Skills, 2003.

CARVAlHO, R. P. Acústica Arquitetônica. 2. ed. Brasília: Thesaurus, 2010.

CHRISTENSEN, C. L.; KOUTSOURIS, G. Odeon Room Acoustics Software, Version 12, User Manual. Universidade Técnica da Dinamarca, 2013.

COHEN, E. Acoustics of Practice Rooms. In: AUDIO ENGINEERING SOCIETY CONVENTION, 92., Wien, 1992. Proceedings... Wien: AES, 1992.

CUSTÓDIO, M. F. Qualidade Sonora Através da Modelagem Numérica e Ensaios Experimentais no Centro de Convenções “Centro Sul” de Florianópolis-SC. Florianópolis, 2004. 157 p. Dissertação (Mestrado em Engenharia Mecânica) - Universidade Federal de Santa Catarina, Florianópolis, 2004.

FIGUEIREDO, F. L. Parâmetros Acústicos Subjetivos: critérios para avaliação da qualidade acústica de salas de música. São Paulo, 2005. 259 f. Dissertação (Mestrado em Musicologia) -Escola de Música, Universidade de São Paulo, São Paulo, 2005.

GADE, A. C. Acoustics in Halls For Speech and Music. In: ROSSING, T. D. (Ed.). Handbook of Acoustics. New York: Springer Science+Business Media, 2007. 
INTERNATIONAL ORGANIZATION FOR STANDARDIZATION. ISO 3382-1: acoustics: measurement of room acoustic parameters: part 1: performance spaces. Switzerland, 2009.

INTERNATIONAL ORGANIZATION FOR STANDARDIZATION. ISO 354: acoustics: measurement of sound absorption in a reverberation room. Switzerland, 2003.

KARSAI, M. The Acoustical Reconstruction of Teaching Studios at the Hungarian Academy of Music. In: INTERNATIONAL CONGRESS ON ACOUSTICS, 8., London, 1974. Proceedings... London: ICA, 1974.

KUTTRUFF, H. Room Acoustics. 5. ed. New York: Spon, 2009.

LAMBERTY, D. C. Music Practice Rooms. Journal of Sound and Vibration, v. 69, n. 1, p. 149-155, 1980.

LANE, R. N.; MIKESKA, E. E. Study of Acoustical, Requirements For Teaching Studios and Practice Rooms in Music School Buildings. Journal of the Acoustical Society of America, v. 27, n. 6, p. 1087, 1955.

LOKKI, T.; SALMENSAARI, O. Report No 71119-1, Measurement Report: Teaching Facilities of Espoo Music Institute. Helsinki, 2007.

LONG, M. Architectural Acoustics. $2^{\text {nd }}$. ed. London: Elsevier, 2011.

NAGATA, M. Nankohall. In: MCCUE, E.; TALASKE, R. H. Acoustical Design of Music Education Facilities. New York, 1989.

QUEIROZ DE SANT’ANA, D.; ZANNIN, P. H. T. Acoustic Evaluation of a Contemporary Church Based on in situ Measurements of Reverberation Time, Definition, and Computer-Predicted Speech transmission index. Building and Environment, v. 46, p. 511-517, 2011.

RINDEL, J. H. The Use of Computer Modeling in Room Acoustics. Journal of VibroEngineering, v. 3, n. 4, 2000.

RYHERD, E. Acoustic Design of Music Rehearsal Rooms. Physics Today, v. 61, n. 8, p. 68-69, 2008.

SÁ, L. R. de. Acústica e Educação em Música: estudo qualitativo para sala de ensaio e prática de instrumento e canto. Curitiba, 2010. $153 \mathrm{f}$. Dissertação (Mestrado em Construção Civil) Escola de Engenharia Civil, Universidade Federal do Paraná, Curitiba, 2010.
SILVA, M. N. P. da. Área da Superfície de Um Corpo Humano. Brasil Escola. Disponível em: $<$ http://brasilescola.uol.com.br/matematica/areasuperficie-um-corpo-humano.htm>. Acesso em: 24 mar. 2016.

TAKAHASHI, V. F. M.; BERTOLI, S. R. Relação Entre Atributos Acústicos e Características Arquitetônicas de Salas de Concerto Aplicando o Método da Síntese da Forma. Ambiente Construído, Porto Alegre, v. 12, n. 1, p. 193-205, jan./mar. 2012.

TENENBAUM, R. A. et al. Hybrid Method For Numerical Simulation of Room Acoustics: part 2: validation of the computational code RAIOS 3.

Journal of the Brazilian Society of Mechanical Sciences Engineering, v. 29, n. 2, p. 222-231, 2007.

TEUBER, W.; VÖELKER E-J. Acoustical Requirements and Results For Music Rehearsal Rooms. In: AUDIO ENGINEERING SOCIETY CONVENTION, 94., Berlin, 1993. Proceedings... Berlin: AES, 1993.

TOPA, M. D. et al. Comparison of Different Experimental Methods for the Assessment of the Room's Acoustics. Acoustical Physics, v. 57, n. 2, p. 199-207, 2011.

VERGARA, E. F. Medições Acústicas e Simulação Computacional de Salas de Aula. In: SCHMID, A. L. Espaços Para Aprender e Ensinar Música: construção e adequação. Brasília: Ministério da Cultura e Ministério da Educação, Coordenação de Aperfeiçoamento do Pessoal de Nível Superior - Capes, Programa PróCultura, Pacto Ambiental, 2013.

VERGARA, E. F.; MARROS, F.; PAUL, S. Caracterização da Qualidade Acústica de Salas de Aula Para Prática e Ensino Musical. Ambiente Construído, Porto Alegre, v. 17, n. 1, p. 23-37, 2017.

VORLÄNDER, M. International Round Robin on Room Acoustical Computer Simulations. In: INTERNATIONAL CONGRESS ON ACOUSTICS, 15., Trondheim, 1995.

Proceedings... Trondheim: ICA, 1995.

VORLÄNDER, M.; SUMMERS, J. Auralization: fundamentals of acoustics, modelling, simulation, algorithms, and acoustic virtual reality. Berlin: Springer, 2008. 
Pollyanna Rodrigues Lima

Departamento de Arquitetura e Urbanismo | Centro Universitário Estácio de Santa Catarina | Av. Leoberto Leal, 431 | São J osé - SC Brasil | CEP 88110-001 | Tel.: (48) 3381-8097 | E-mail: pollyannalima@outlook.com

\section{Erasmo Felipe Vergara}

Laboratório de Vibrações e Acústica, Departamento de Engenharia Mecânica | Universidade Federal de Santa Catarina | Campus Universitário, Trindade | Caixa Postal 476 | Florianópolis - SC - Brasil | CEP 88040-970 | Tel.: (48) 3721-9227 Ramal 228 | E-mail: e.f.vergara@ufsc.br

\section{Revista Ambiente Construído}

Associação Nacional de Tecnologia do Ambiente Construído

Av. Osvaldo Aranha, 99 - 3o andar, Centro

Porto Alegre - RS - Brasil

$$
\text { CEP 90035-190 }
$$

Telefone: +55 (51) 3308-4084

Fax: +55 (51) 3308-4054

www. seer. ufrgs. br/ ambienteconstruido

E-mail: ambienteconstruido@ufrgs.br 